\title{
An analysis of psychiatric referrals In a multidisciplinary General hospital in Sri Lanka
}

Dharmawardene V.

Department of Psychiatry Teaching Hospital Anuradhapura

\section{Introduction}

Psychiatric morbidity significantly affects the outcome of physical illness. Rate of psychiatric morbidity is $20-50 \%$ of the inpatient population in global studies. There is limited published data on the subject from a Sri Lanka.

\section{Objective}

To describe the patterns of psychiatric morbidity in referrals from the inpatient population received at the psychiatric unit of teaching hospital Anuradhapura, a multidisciplinary hospital.

\section{Methods and design}

A Retrospective file review was carried out into all the ward referrals received between 01 May 2012 and 31 July 2012. All the assessments during the period had been conducted by one consultant psychiatrist.

\section{Results:}

There were 213 Referrals received during the study period comprising $0.58 \%$ of the admissions. $59 \%$ were females. Highest number was received from the general medical wards $(47 \%)$ followed by cardiology (18\%) and surgery (12\%). 30\% did not have any diagnosable psychiatric illness. Commonest psychiatric diagnosis was depressive disorder (19\%). 24\% of the referrals were for patients who have attempted self harm (DSH). Oleander seed ingestion was the commonest method $(44 \%)$ followed by medication overdose $(22 \%)$ with only $18 \%$ seen with ingestion of agrochemicals. $11 \%$ patients who have attempted self harm had depressive illness. The rate was 06 $\%$ for oleander seed ingestion.

\section{Conclusions:}

Though Depressive illness appears to be the commonest diagnosis, it is relatively less common among the DSH patients. DSH by agrochemicals are represented less in this sample. 